\title{
Multi-messenger aspects: Composition, propagation, \& acceleration
}

\author{
Andrew M. Taylor ${ }^{\mathrm{a}}$ \\ Dublin Institute for Advanced Studies, 31 Fitzwilliam Place, Dublin 2, Ireland
}

\begin{abstract}
Using recent Pierre Auger Observatory results on the UHECR spectrum and composition, the requirements placed on the sources of these particles are discussed. In this sense, the author interprets the term "multi-messenger" throughout as to refer to the additional information provided by the composition. The spatial distribution of these sources is investigated along with the energy distribution of UHECR they output. These investigations reveal the need for local UHECR sources which output a hard spectrum of intermediate/heavy UHECR. These results demand that local $(<80 \mathrm{Mpc})$ UHECR sources exist, placing exciting and difficult requirements on the local extragalactic candidate sources. Angular correlation studies in collaboration with composition information also demonstrated to offer great potential for isolating the UHECR source distance for which an angular clustering of events is found. Specifically, for the case a correlation of the 13 events correlated with the direction of Cen A, it is shown that the composition information, and specifically, the lack of a lower energy proton correlation, can potentially constrain the source distance to be less than $15 \mathrm{Mpc}$. The fragility of nuclei with accelerators are also used to place constraints are on the source environment. These constraints motivate $0.1-1000 \mathrm{mG}$ strength magnetic fields exist within the source and that quasi-relativistic scatterers are also present. A specific example of a diffuse large scale "nuclei friendly" accelerator, which meets the outline constraints is put forward.
\end{abstract}

\section{Introduction}

The revelation following recent Pierre Auger Observatory (PAO) results that ultra high energy cosmic rays (UHECR) possess a significant nuclei component, as shown in Fig. 1, has important astrophysical implications. Leaving the question of the available relative abundance of these species in the source region aside, the question of their acceleration and transport are here addressed.

Using the extra degree of information provided by the particle composition, on top of that already provided by the energy and direction of the UHECR, the consequences of this result are discussed. Firstly, the source injection spectra and composition consistent with these results is summarised. The subsequent constraints placed on the locality of these sources is also addressed. Methods employing this new composition information in order to locate the sources highlighted. Finally, an example of a "nuclei friendly" acceleration site is provided, with generalities from this example being noted where possible.

\section{Propagation physics and description}

Before their arrival, UHECR must propagate across astronomical distances between their sources and Earth. The arriving flux from an ensemble of their sources is naturally expected to contain a suppression feature just below the highest energies currently observed. Such a feature results as a consequence of UHECR interactions with background photons. For the case of a proton dominated UHECR composition, photo-pion production interactions will rapidly start to dominate energy losses

\footnotetext{
a e-mail: taylora@cp.dias.ie
}

at energies just below $10^{20} \mathrm{eV}$, leading to a suppression feature being expected at these energies, referred to as the Greisen-Zatsepin-Kuz'min (GZK) cutoff [3,4].

The expectation of a high energy suppression feature, however, is not unique to the proton composition scenario. Indeed a similar feature is also naturally expected for the case when a significant fraction of the population consists of nuclei. However, for such a scenario, it is instead photo-disintegration interactions which lead to the suppression in flux at the highest energies. Subsequently, the identification of a suppression feature at the highest energies is unable to provide much clue as to the underlying source composition.

In order to get a more developed understanding to allow the composition measurement results to be interpreted, the energy loss lengths for different species of UHECR nuclei must be calculated. The calculation of these loss rates follow the general formula,

$$
R_{A, \gamma}=\frac{1}{2 \Gamma_{A}^{2}} \int_{0}^{\infty} \frac{1}{\epsilon_{\gamma}^{2}} \frac{d n_{\gamma}}{d \epsilon_{\gamma}} d \epsilon_{\gamma} \int_{0}^{2 \Gamma_{A} \epsilon_{\gamma}} \epsilon_{\gamma}^{\prime} \sigma_{A \gamma}\left(\epsilon_{\gamma}^{\prime}\right) K_{A \gamma} d \epsilon_{\gamma}^{\prime}
$$

where $d n_{\gamma} / d \epsilon_{\gamma}$ describes the spectral shape of the target photons being interacted with, $\sigma_{A \gamma}$ dictates the interaction rate of the UHECR with these target photons, and $K_{A \gamma}$ is the fractional energy loss of the UHECR as a result of the interaction. Example energy loss rate curves for different species are given in reference [5].

Using a complete set of such loss/interaction rate curves for the ensemble of nuclear species, the transmutation of species as they propagate through extragalactic radiation fields can be calculated. A Monte Carlo implementation for calculating the temporal evolution of the 

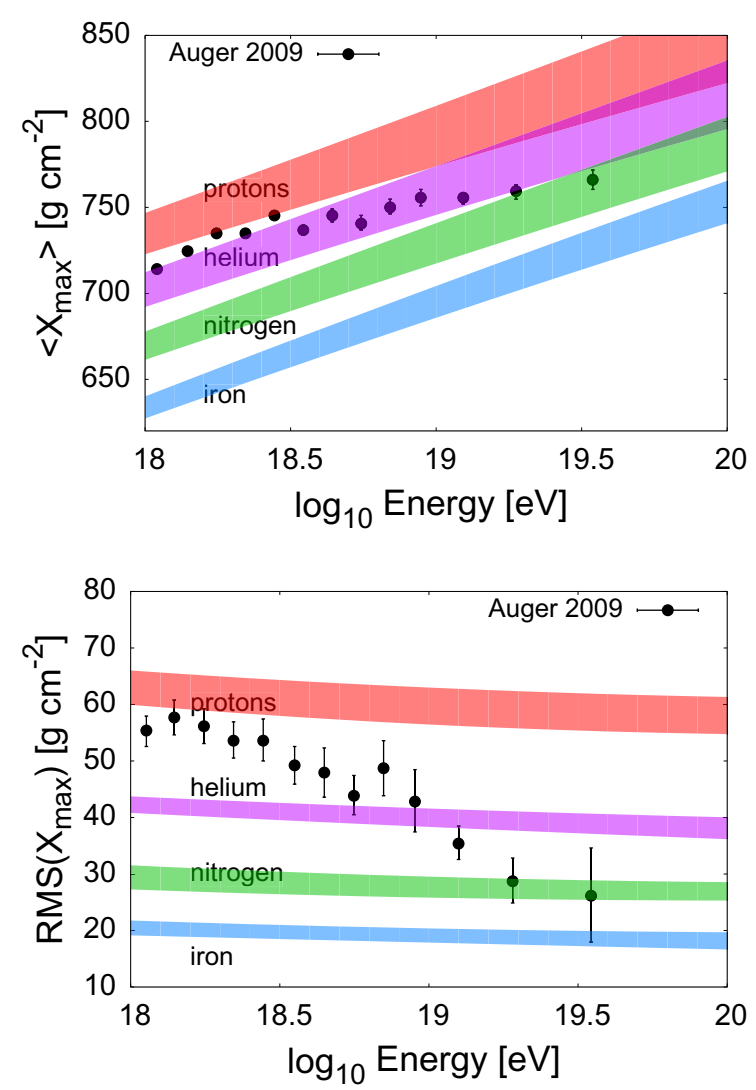

Figure 1. Plots showing PAO measurements of the composition sensitive quantities $\left\langle X_{\max }\right\rangle$ and $\operatorname{RMS}\left(X_{\max }\right)$.

nuclear state populations was employed in reference [5]. For these calculations, all possible decay routes through a whole network of possible alternative nuclear isotope states were considered.

\section{Source proximity}

In order to keep the situation of the UHECR propagation as simple as possible, the presence of extragalactic magnetic fields on UHECR propagation are neglected. Furthermore, with no prior knowledge about the UHECR source population, a "universal" (homogeneous and isotropic) distribution is generally assumed. We adopt this assumption here as a means of investigating signatures of a departure from it. In order to quantify the effect of a different source distribution in this paper, we separate out the fluxes produced from source regions with shells of radii 0-3 Mpc, 3-9 Mpc, 9-27 Mpc, 27-81 Mpc, and 81-243 Mpc surrounding the Earth, as depicted in the left-panel of Fig. 2. In this way, the results obtained may be used to encapsulate the effects introduced by a non"universal" local void of UHECR sources.

As was found in previous work [6,7], the contour plots reveal that sources emitting intermediate-heavy compositions $(A>20)$ UHECR with hard spectral indices $(\alpha \lesssim 2)$ and intermediate energy cutoffs $\left(E_{\mathrm{Fe}, \max } \sim\right.$ $10^{21} \mathrm{eV}$ ) are best able to describe the current data. It should also be noted that for light-nuclei type sources the contour space was found to be considerably diminished, with no
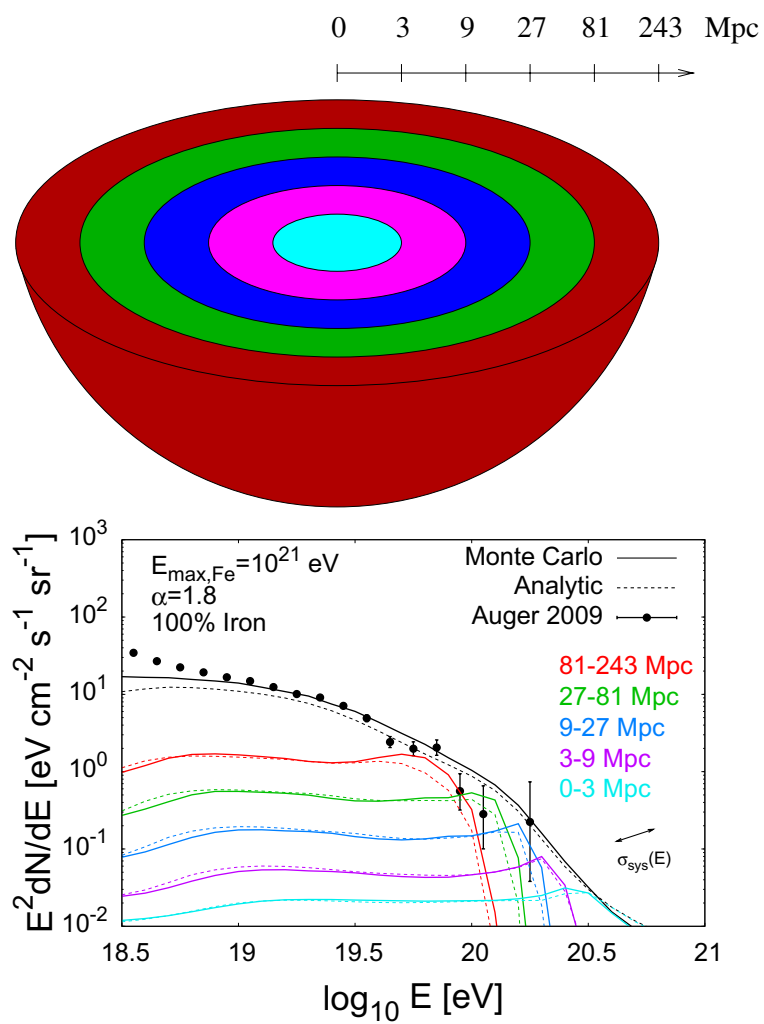

Figure 2. Left-panel: a depiction of the source shells considered. Right panel: a spectral plot showing PAO measurements, Monte Carlo and analytic derived fluxes arriving from the different source shells.

good-fit contours existing for a proton-only scenario, even at the $99 \%$ C.L.

Interestingly, the introduction of a minimum distance to the first source can dramatically alter the good-fit contours. In particular, for the shell sizes considered, it is seen that for source emitting either silicon or iron type nuclei compositions, the 99\% C.L. contours undergo a rapid decrease in size for minimum source distances in the range $9-27 \mathrm{Mpc}$ and $27-81 \mathrm{Mpc}$ respectively. Furthermore, these plots also indicate that for minimum source distances beyond these constrained ranges, uncomfortably large cutoff energies, $E_{\mathrm{Fe} \text {,max }}$, are required by the source population. This result encapsulates one of the key findings from this study, that sources of hard spectra UHECR nuclei sources with local distances, $\sim 40-80 \mathrm{Mpc}$ must exist in local extragalactic space.

Indeed, the realisation that hard spectra are required in order to explain the observed UHECR flux and composition results, collectively, has itself motivated several studies to find a natural explanation for such spectra. In [8], a study was carried out which utilised the magnetic horizon to introduce such hard spectra through propagation effects. Though strong $\mathrm{nG}$ strength extragalactic magnetic fields were found to indeed be sufficient to introduce such effects, the motivation of such strong fields with significant filling factors brings into question the validity of such a solution to the "hard spectra problem" [9]. Though a clear resolution of this problem still remains, the challenge it presents almost invariably 
provides us with clues as to the nature of UHECR sources. In the following section, a discussion of how correlation signatures in collaboration with composition information can also probe local sources is provided.

\section{Correlation with sources}

Both the PAO and Telescope Array (TA) instruments have reported large scale $\left(\sim 20^{\circ}\right)$ anisotropy/clustering signatures $[11,12]$ whose statistical significance have increased through consecutive years worth of data. Though the astrophysical object class with which this correlation remains unclear, their general proximity is guaranteed by the energy cut $E>57 \mathrm{EeV}$ which was adopted in both analyses. With X-ray bright AGN remaining one of the few non-thermally bright local emitters, a comparison of the UHECR event distribution with these AGN is adopted. Figure 3 shows the distribution of X-ray bright AGN overlaid onto the anisotropy signal regions recently discovered.

A further probe of the source from which such anisotropy signals originate is potentially also available using the composition information. Unless extragalactic fields are $\mathrm{nG}$ in strength, the CR deflection away from the source direction at very high energies is expected to be dominated by the Galactic magnetic field structure. Thus, adopting a specific composition, the deflections experienced may be taken into account and the intrinsic angular distribution arriving to the edge of the Galactic magnetic field structure may be determined from that observed. Alternatively, for a given candidate source, the angular deflection size expected to be introduced by the Galactic magnetic field distortions of the source image may be inferred, in a source composition dependent manner. This second approach was used in [13] for the case of an association of 13 PAO events with the radio galaxy Cen A. Assuming such an association of these events with Cen $\mathrm{A}$ is genuine, the UHECR composition of the events was demonstrated to need to be light $(A<14)$ in order that the Galactic magnetic fields do not disperse too broadly the arriving CR from this object.

More generally, an anisotropy signal seen for a specific species, should be repeated at similar rigidities by other species emitted by the source [14]. Indeed, even if the source does not emit lighter particles, such species will invariably be produced en-route through photodisintegration processes [15]. In this way, future anisotropy signals using composition information can be used to provide a probe on the source distance information. Such analysis, however, will first require accurate shower-byshower composition information to be available.

As an example case to demonstrate the potential of such a probe, we consider once again the 13 events arriving from the direction of Cen A. Assuming an Oxygen composition for these events and coupling this information with the lack of lower energy proton anisotropy output by the source/produced en-route, place a maximum distance to the source of $15 \mathrm{Mpc}$ [13]. Furthermore, the required metallicity within the source acceleration zone suggested is surprisingly high, which itself might provide new insight into the nature of the acceleration site.

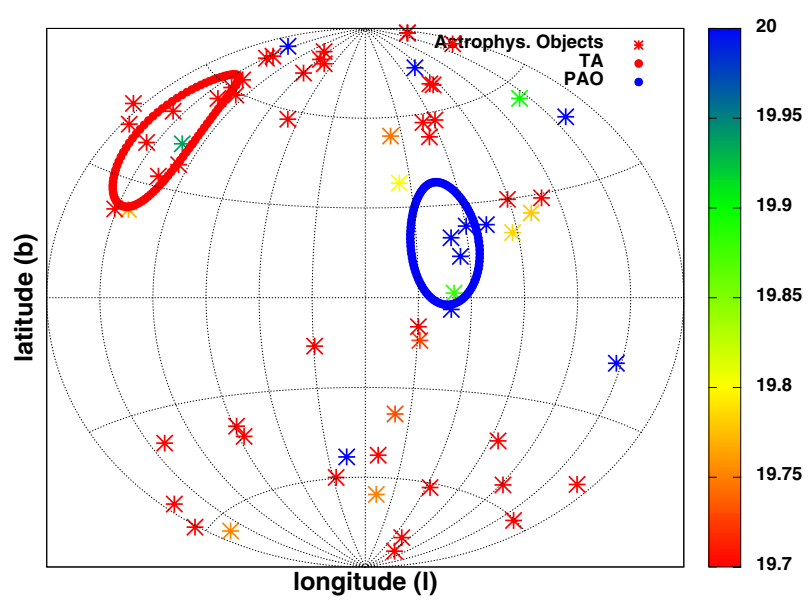

Figure 3. The distribution of non-thermal locally bright $\left(L_{X}>\right.$ $10^{42} \mathrm{erg} \mathrm{s}^{-1}$ ) AGN in Galactic coordinates. The AGN brightness, $s$, given in the scale is $s=\log _{10}\left(L_{X} / z^{2}\right)-27.5$, where $z$ is the AGN redshift. The $20^{\circ}$ circles shown highlight regions in which $\mathrm{CR}$ over-densities have been observed, $\left(-46.4^{\circ}, 17.7^{\circ}\right)$ for PAO [11] and $\left(146.7^{\circ}, 43.2^{\circ}\right)$ for TA [12].

\section{Candidate sources}

The actual sources of UHECR has been a long sought for aim of cosmic ray studies. Despite the considerable time that has passed since their discovery, the sources of these high energy particles remains unclear. The "Hillas criterion" [10] for a candidate source demands that $E_{\max }=$ $\beta_{s} R_{\mathrm{s}} Z e B_{\mathrm{s}}$, where $E_{\max }$ is the maximum energy particle that may be accelerated by a source of size $R_{\mathrm{S}}$ containing magnetic fields of strength $B_{\mathrm{s}}$ and an isotropic distribution of internal scatters moving at velocity $\beta_{s} c$. Applied to $10^{20} \mathrm{eV}$ nuclei, the $R_{\mathrm{s}}-B_{\mathrm{s}}$ parameter space quickly reduces viable sources to just a few potential candidates, whose scatters must be moving at velocities close to $c$. This constraint may be written as

$$
\beta_{s} Z\left(\frac{R_{s}}{\mathrm{kpc}}\right)\left(\frac{B_{s}}{0.1 \mathrm{mG}}\right)>1 .
$$

Such considerations for source requirements present necessary but not sufficient conditions for an object to be considered a viable candidate UHECR source. On top of these demands, the radiative loss rates as well as the acceleration rates available within the sources must be taken into account. The maximal acceleration timescale, assuming this occurs at the Bohm limit, is

$$
t_{\text {acc. }}=\frac{E}{Z e c B_{s} \beta_{s}^{2}}=\frac{1}{2 \pi} \frac{\Gamma}{\beta_{s}^{2}} \frac{h}{M c^{2}}\left(\frac{B_{\text {crit. }}}{B_{\mathrm{s}}}\right) .
$$

Similarly, the synchrotron loss time is

$$
t_{\text {loss }}^{\text {sync. }}=\frac{9}{(8 \pi \alpha)} \frac{h}{E}\left(\frac{B_{\text {crit. }}}{B_{\mathrm{s}}}\right)^{2},
$$

where $B_{\text {crit }}=\left(M^{2} / Z m_{e}^{2}\right) B_{\text {crit }}^{e}$, with $B_{\text {crit }}^{e} \approx 4.4 \times 10^{13} \mathrm{G}$. Thus, if the acceleration time equals the synchrotron loss time,

$$
E_{\max }=\frac{3 \beta_{s}}{2 \alpha^{1 / 2}}\left(\frac{B_{\text {crit. }}}{B_{\mathrm{s}}}\right)^{1 / 2} M c^{2} \text {. }
$$


This places an extra constraint on the velocity of the scatterers present within the accelerator. Applied once again to sources of $10^{20} \mathrm{eV}$ UHECR,

$$
\left(\frac{\beta^{2} A^{4}}{Z^{3}}\right)\left(\frac{0.5 \mathrm{G}}{B_{s}}\right)>1 .
$$

Finally, losses through pion-production/photo-disintegration interactions, expected to dominate energy losses of the highest energy UHECR protons and nuclei, respectively, also place constraints on the source parameters. Approximating the loss time to $\sim \mathrm{Myr}$, valid for energies above $10^{20} \mathrm{eV}$, a final constraint on the source parameters is

$$
Z \beta_{s}^{2}\left(\frac{B_{s}}{0.1 \mathrm{mG}}\right)>1 .
$$

The above results indicate that few candidate objects are able to satisfy the difficult constraints on the acceleration environment, with $0.1-1000 \mathrm{mG}$ strength magnetic fields and fast/mildly relativistic scatterers needed to be present in the acceleration environment for optimal acceleration. Furthermore, these constraints can be considered conservative since they neglect the fact that UHECR need also survive the extragalactic environment during their propagation from source to Earth. Interestingly, both these requirements and those obtained previously from the source proximity study may be met in certain regions around local AGN. In the following section a specific example AGN acceleration environment is focused on.

\section{Example "nuclei friendly" acceleration}

Following on from the discussion in the previous section on the general source requirements such that $\mathrm{CR}$ nuclei can be accelerated successfully to UHE, the case of a specific source scenario which meets these requirements is discussed.

In general, compact acceleration sites appear excluded through energy loss arguments. On the contrary, the acceleration by large diffuse accelerators present certain advantages provided that the slow acceleration rates associated with such sites may be overcome. Indeed, for sufficiently fast Alfvén waves, the stochastic acceleration of protons up to $10^{19} \mathrm{eV}$ may operate such that energy losses do not hinder the process. Furthermore, the production of UHECR on large diffuse scales helps alleviate adiabatic loss problems associated with compact accelerators and the subsequent $\mathrm{CR}$ escape into extragalactic space.

Focusing on nearby FR-1 galaxies such as Cen A, such an acceleration mechanism is in fact quite plausible, provided the specific conditions required for the source environment are first met. Primarily, it is required that the lobe's densities be orders of magnitude below the present upper-bound values currently in place. Such a requirement is necessary in order that very fast Alfvén speeds exist within this region. Interestingly, recent observations indicate that mildly relativistic Alfvén speeds may indeed exist within the radio Galaxy environment [17].

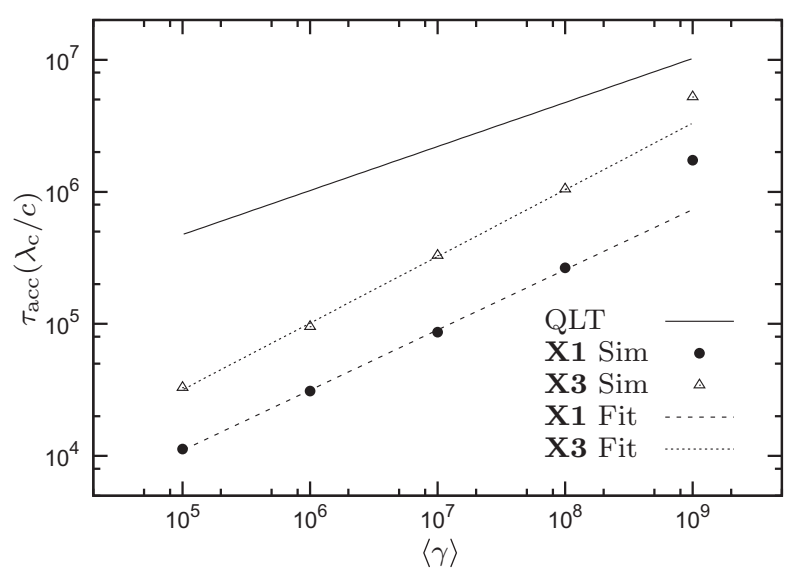

Figure 4. A comparison with expectations from quasi-linear theory (QLT) of the acceleration times obtained from a simulation of particle scattering off isotropic magnetic field turbulence in radio lobes [16]. The labels $\mathbf{X} \mathbf{1}$ and $\mathbf{X} \mathbf{3}$ refer to 1-dimensional and 3-dimensional simulation setups, respectively.

Beyond the fast scatterer assumption, only acceleration sites "distant" from the host galaxy possess radiation fields sufficiently weak that secondary losses can be safely neglected. It is also assumed that magnetic wave turbulence exists throughout the lobe region, with wavelengths ranging from $\lambda_{\min }=10^{-5} \mathrm{pc}$ to $\lambda_{\max }=1 \mathrm{kpc}$, with a Kolmogorov spectrum of amplitudes.

With the above outlined setup, the acceleration of UHECR by Alfvén waves via the second-order Fermi process in the lobes of giant radio galaxies was shown to be viable [16]. The attempt of the UHECR population to thermalise with the giant macroscopic waves present, which propagated isotropically with Alfvén speed velocities throughout the lobes, was demonstrated explicitly. A comparison to the rates obtained with expectations from quasi-linear theory is shown in Fig 4. A factor of order a few faster acceleration rates were found to be obtained in the numerical simulations.

\section{Conclusion}

The recent Pierre Auger Observatory spectrum composition results places interesting new requirements on the UHECR sources. From the studies discussed here, the need for local UHECR sources which output a hard spectrum of intermediate/heavy UHECR was revealed. This requirement was found with the added condition that local $(<80 \mathrm{Mpc})$ UHECR sources exist. The angular correlation of UHECR in collaboration with their composition information was also shown to hold great potential for providing insight into the third dimension of such correlations, allowing bounds to be placed on the UHECR source distance.

With regards to the actual sources of these particles, it is highlighted that the Hillas condition for UHECR acceleration is only a "necessary but not sufficient" requirement for UHECR acceleration [10]. Beyond this constraint, the requirement that candidate acceleration sites simultaneously both provide sufficiently fast acceleration before escape and at the same time do not possess 
an intense radiation field environment destructive to nuclei during their acceleration provides some challenge in order to be satisfied. It is highlighted that local AGN are able to satisfy these conditions. To elucidate this point, an example acceleration site around AGN is provided, in the giant radio lobe structures inflated by AGN jets.

Though the case for the presence of nuclei in the arriving UHECR is still not water tight, the mounting evidence presently available, pointing in this direction, motivates a deeper consideration of the major consequences of such a result. As demonstrated here, these consequences severely restrict arguments about source candidates, providing crucial new information on their potential origins.

A.T. acknowledges a Schröedinger fellowship at DIAS.

\section{References}

[1] M. Unger, f. t. P. A. Collaboration, arXiv:1103.5857 [astro-ph.HE]

[2] J. Abraham et al. [Pierre Auger Collaboration], Phys. Rev. Lett. 104, 091101 (2010)

[3] K. Greisen, Phys. Rev. Lett. 16, 748 (1966)

[4] G. T. Zatsepin and V. A. Kuz'min, JETP Lett. 4, 78 (1966)

[5] D. Hooper, S. Sarkar and A. M. Taylor, Astropart. Phys. 27199 (2007) [astro-ph/0608085]
[6] D. Hooper and A. M. Taylor, Astropart. Phys. 33, 151 (2010)

[7] A. M. Taylor, M. Ahlers and F. A. Aharonian, Phys. Rev. D 84 (2011) 105007 [arXiv:1107 . 2055 [astroph.HE]]

[8] S. Mollerach and E. Roulet, JCAP 1310 (2013) 013 [arXiv:1305.6519 [astro-ph.HE]]

[9] R. A. Batista and G. Sigl, arXiv:1407.6150 [astroph.HE]

[10] A. M. Hillas, Ann. Rev. Astron. Astrophys. 22, 425 (1984)

[11] P. Abreu et al. [Pierre Auger Collaboration], Astropart. Phys. 34 (2010) 314 [arXiv:1009.1855 [astro-ph.HE]]

[12] R. U. Abbasi et al. [Telescope Array Collaboration], Astrophys. J. 790 (2014) L21 [arXiv:1404.5890 [astro-ph.HE]]

[13] R. Y. Liu, X. Y. Wang, W. Wang and A. M. Taylor, Astrophys. J. 755 (2012) 139 [arXiv:1206.3907 [astro-ph.HE]]

[14] M. Lemoine and E. Waxman, JCAP 0911 (2009) 009 [arXiv:0907.1354 [astro-ph.HE]]

[15] R. Y. Liu, A. M. Taylor, M. Lemoine, X. Y. Wang and E. Waxman, Astrophys. J. 776 (2013) 88 [arXiv:1308.5699 [astro-ph.HE]]

[16] S. O'Sullivan, B. Reville and A. M. Taylor, Mon. Not. Roy. Astron. Soc. 400 (2009) 248 [arXiv:0903. 1259 [astro-ph.HE]]

[17] G. Lapenta and P. P. Kronberg, Astrophys. J. 625 (2005) 37 